\title{
Ácidos graxos trans: implicações nutricionais e fontes na dieta
}

\author{
Trans fatty acids: nutritional implications \\ and sources in the diet
}

Clayton Antunes MARTIN ${ }^{1}$

Makoto MATSHUSHITA²

Nilson Evelázio de SOUZA²

\section{R E S U M O}

Este artigo revisa as principais fontes de ácidos graxos trans na dieta e as implicações nutricionais da ingestão elevada destes isômeros. São apresentados resumidamente os métodos analíticos utilizados na identificação e quantificação dos ácidos graxos trans, sendo abordados as suas vantagens e desvantagens. Os alimentos que empregam gordura parcialmente hidrogenada na sua produção, são fontes importantes de isômeros trans na dieta da maior parte da população em países industrializados. Este estudo compara os níveis de ácidos graxos trans em gorduras hidrogenadas, margarinas e batatas frita, analisados em diversos países, incluindo o Brasil. Esta avaliação indica a presença de níveis elevados de isômeros trans em alimentos produzidos no Brasil.

Termos de indexaç̧ão: ácidos graxos trans, doenças cardiovasculares, fontes na dieta, dieta.

\section{A B S T R A C T}

This article review the main sources of trans fatty acids in the diet and nutritional implications of the high intake of these isomers. Analytical methods for the identification and quantification of trans fatty acids are presented briefly with regard to advantages and drawbacks of each method. Foods make with partially hidrogenated fats are important sources of trans isomers in the diets of most people in industrialized countries. It is made a comparison between levels of trans fatty acids in shortenings, margarines and potato chips evaluated in Brazil and in other countries. High levels of trans isomers are noted in Brazilian foods.

Index Terms: trans fatty acids, coronary heart disease, sources, diet.

\footnotetext{
1 Pós-Graduação em Química, Universidade Estadual de Maringá. Maringá, PR, Brasil.

2 Departamento de Química, Universidade Estadual de Maringá. Av. Colombo, 5790, 87020-900, Maringá, PR, Brasil. Correspodência para/Correspondence to: N.E. SOUZA. E-mail: nesouza@uem.br
} 


\section{N T R O D U Ç Ã O}

A função dos óleos e gorduras na nutrição humana tem sido intensamente pesquisada e discutida nas últimas décadas. Como resultado, vem sendo enfatizado a importância da ingestão de ácidos graxos $\omega-3$, a redução de ácidos graxos saturados e mais recentemente, o controle da ingestão de ácidos graxos trans.

Os ácidos graxos são denominados trans, quando os hidrogênios ligados aos carbonos de uma insaturação encontram-se em lados opostos. Na natureza, os ácidos graxos geralmente são encontrados na configuração cis. Nesta configuração, os hidrogênios ligados aos carbonos da dupla ligação se encontram do mesmo lado.

Os ácidos graxos trans (AGT) sempre estiveram presentes na alimentação humana, através do consumo de alimentos provenientes de animais ruminantes. Entretanto, com a produção de substitutos para a manteiga e as gorduras animais, principalmente a partir da hidrogenação parcial de óleos vegetais, cuja presença destes isômeros na dieta se tornou significativa'.

A produção de gordura vegetal hidrogenada no Brasil, começou por volta de 1960. Nos últimos anos, a indústria nacional de gorduras hidrogenadas, esteve mais direcionada para o desenvolvimento de produtos com características específicas, que atendessem às necessidades da indústria de alimentos, do que para a produção de gorduras com baixos níveis de isômeros trans².

Neste estudo, são abordados as principais implicações nutricionais da ingestão elevada de ácidos graxos trans e analisados os dados sobre os teores destes isômeros em alguns alimentos industrializados, que foram avaliados em diversos países, incluindo o Brasil.

\section{M P LICAÇÕ ES NUTRICIONAIS}

Em 1961, já se realizavam estudos comparando os efeitos sobre os níveis de colesterol plasmático da ingestão de gordura parcialmente hidrogenada, de óleos vegetais e gorduras saturadas. Estes estudos mostraram os níveis de colesterol total associados à ingestão de gordura saturada eram um pouco mais elevados que os níveis relacionados a gordura parcialmente hidrogenada ${ }^{3,4}$.

Somente em 1990, através de um estudo realizado por Mensink \& Katan ${ }^{5}$, a atenção de muitos pesquisadores foi despertada para a investigação dos efeitos adversos dos ácidos graxos trans. Neste estudo, os autores mostraram que a ingestão elevada de AGT aumentava os níveis da lipoproteína de baixa densidade (LDL-C) de maneira similar aos ácidos graxos saturados. Entretanto, foi observado que os AGT reduziam os níveis da lipoproteína de alta densidade $(\mathrm{HDL}-\mathrm{C})$, alterando significativamente a razão entre a LDL-c e a HDL-c, em relação à dieta em que os AGT foram substituídos por ácidos graxos saturados.

Este efeito dos AGT sobre os níveis plasmáticos da LDL-c e da HDL-c, tem sido confirmado em vários estudos ${ }^{6-11}$, realizados com diferentes porcentagens de AGT (0,5-11) em relação a energia total da dieta ingerida. Ao analisar alguns destes estudos, Ascherio et al. ${ }^{12}$ sugeriram que a elevação em $2 \%$ na ingestão de ácidos graxos trans pode estar relacionada a um aumento de 0,1 na relação LDL-C/HDL-c. Tem sido observado que o aumento de uma unidade $(1,0)$ nesta relação está associado a elevação em cerca de $53 \%$ do risco de doenças cardiovasculares ${ }^{13}$.

Os ácidos graxos trans vem sendo associados com o aumento dos níveis de triacilgliceróis no plasma sanguíneo ${ }^{4,12}$. Este efeito tem sido observado através da substituição de ácidos graxos com a configuração cis por AGT, em uma mesma dieta. Hu et al. ${ }^{14}$ sugeriram uma provável contribuição deste efeito na elevação do risco de doenças cardiovasculares. Entretanto, alguns autores ${ }^{9,15}$ não tem verificado diferenças significativas entre os níveis de triacilgliceróis avaliados. 
Diversos estudos $5,8-11,16,17$ tem avaliado a influência da ingestão elevada de ácidos graxos trans sobre os níveis da lipoproteína(a) (Lp(a)), considerada um fator de risco para doenças cardiovasculares. Segundo Lippi \& Guidi ${ }^{18}$, a Lp(a) provavelmente atua inibindo competitivamente o plasminogênio, o que impossibilita a sua ativação em plasmina, uma enzima responsável pela degradação da fibrina.

Os níveis da $L p(a)$ variam entre indivíduos, devido principalmente a fatores genéticos ${ }^{19}$, mas tem sido verificado uma alteração moderada em função da composição em ácidos graxos da $\operatorname{dieta}^{20}$. Alguns dos estudos realizados $5,8,16,21$ indicaram um aumento significativo dos níveis desta lipoproteína, quando diferentes teores de ácidos graxos saturados foram substituídos por ácidos graxos trans. O efeito da intensidade deste aumento sobre o risco de doenças cardiovasculares ainda é desconhecido, mas parece ter maior impacto em indivíduos geneticamente condicionados a ter níveis maiores de $L p(a)^{16}$.

Estudos realizados em cobaias ${ }^{22-24}$ tem mostrado a competição de ácidos graxos trans com ácidos graxos das famílias $\omega$-6 e $\omega$-3, nas reações de dessaturação e elongação da cadeia, resultando na formação de eicosanóides sem atividade biológica. Além disso, os AGT monoinsaturados e poliinsaturados podem inibir as enzimas $\beta 6$ e $\beta 5$ dessaturase, bloqueando o metabolismo dos ácidos graxos essenciais. Tem-se sugerido a ocorrência deste processo em humanos, com destaque para o impacto na fase gestacional, ao alterar o desenvolvimento intra-uterino pela inibição da síntese dos ácidos araquidônico e docosa-hexaenóico ${ }^{25}$. Outra possível consequência deste processo é a alteração no balanço existente entre prostaglandinas $e$ tromboxanos, o que pode favorecer a agregação plaquetária, contribuindo para o desenvolvimento de aterosclerose ${ }^{14}$.

Contudo, a investigação da influência dos AGT sobre a atividade das enzimas dessaturases no homem, ainda é escassa. Em recente estudo realizado com homens de idade entre 30 e 50 anos, Scrimgeour et al. ${ }^{26}$ verificaram que a ingestão de isômeros trans do ácido $\alpha$-linolênico como $0,6 \%$ da energia total, não alterou a conversão do ácido linoléico em ácido araquidônico em relação a dieta em que estes isômeros foram reduzidos a cerca de 0,1\% da energia ingerida. Embora este resultado tenha sido obtido com níveis de ingestão de isômeros trans considerados baixos, este estudo sugere que dietas com teores reduzidos de AGT não influenciam a atividade das enzimas $\beta 5$ e $\beta 6$ dessaturase em adultos.

O aumento do consumo de alimentos contendo níveis elevados de AGT, pode além das implicações nutricionais apresentadas, ter como consequência direta a redução da ingestão de ácidos graxos essenciais, favorecendo o desenvolvimento de síndromes relacionadas a deficiência destes ácidos graxos.

\section{A N ÁLISE DE ÁCIDOS GRAXOSTRANS}

Na determinação dos ácidos graxos trans em alimentos, a cromatografia gasosa, a cromatografia em camada delgada impregnada com nitrato de prata e a espectrofotometria no infravermelho são as técnicas mais empregadas ${ }^{27}$.

A análise por cromatografia gasosa emprega colunas capilares com fase estacionária de elevada polaridade, que possibilitam a separação dos isômeros cis e trans ${ }^{28}$.

A cromatografia em camada delgada impregnada com nitrato de prata $\left(\mathrm{CCD} / \mathrm{Ag}^{+}\right)$está baseada na capacidade que o íon prata tem em formar complexos com ácidos graxos insaturados $^{29}$. A associação entre a $\mathrm{CCD} / \mathrm{Ag}^{+}$e a cromatografia gasosa permite obter uma maior eficiência na separação dos isômeros cis e trans, em relação a análise realizada somente por cromatografia gasosa. O aspecto desfavorável deste método é o elevado tempo de análise e a impossibilidade de automação ${ }^{30}$.

A determinação dos isômeros trans através da espectrofotometria no infravermelho está 
fundamentada na absorção característica das ligações trans em $967 \mathrm{~cm}^{-1}$ e na sua correlação com o ácido elaídico ${ }^{27}$. Este método é rápido e de fácil execução, entretanto não fornece informações sobre as proporções dos diferentes isômeros, o números de insaturações e as suas posições. Os níveis de ácidos graxos trans determinados por esta técnica são geralmente maiores que os obtidos por CCD/Ag ${ }^{+}$associada a cromatografia gasosa. Alguns autores $27,31,32$ atribuem este aumento à presença de ácidos graxos poliinsaturados que apresentam mais de uma insaturação com a configuração trans e aos isômeros conjugados, que tem absorção característica, próxima da região de absorção dos isômeros trans.

A avaliação de isômeros trans por espectrofotometria no infravermelho tem sido associada a análise por cromatografia gasosa, possibilitando a obtenção de resultados semelhantes aos determinados por CCD/Ag+ associada à cromatografia gasosa ${ }^{31,33}$.

\section{FONTES DEÁCIDOS GRAXOS TRANS NA DIETA}

Os ácidos graxos trans presentes na dieta são oriundos de gorduras parcialmente hidrogenadas, de óleos refinados, da carne, leite e derivados de animais ruminantes. Segundo Larqué et al. ${ }^{34}$ os alimentos contendo gordura parcialmente hidrogenada contribuem com cerca de $80 \%$ a $90 \%$ da ingestão diária de AGT. Para alimentos provenientes de animais ruminantes esta contribuição é bem menor, sendo estimada em torno de $2 \%$ a $8 \%$. Os óleos refinados apresentam níveis razoavelmente pequenos $(1,0-1,5 \%)$ de $A G T$, mas a reutilização, principalmente no preparo de alimentos fritos, pode tornar significativa a sua contribuição na ingestão diária de $A G T^{35,36}$.

O grande interesse em utilizar gorduras hidrogenadas na produção de alimentos deve-se ao desenvolvimento de gorduras cada vez mais específicas, com o objetivo de melhorar as características físicas e sensoriais dos alimentos.
No Brasil, a utilização de gorduras hidrogenadas é ampla e muitas vezes indiscriminada, envolvendo a produção de margarinas, cremes vegetais, pães, biscoitos, batatas fritas, massas, sorvetes, pastéis, bolos, entre outros alimentos ${ }^{37}$.

Inúmeros estudos tem sido conduzidos em diversos países para determinar os teores de AGT em alimentos produzidos em indústrias, confeitarias e redes de fast-food (Tabela 1), objetivando avaliar as diversas fontes da dieta e estimar a ingestão diária dos AGT.

A análise dos dados de AGT (Tabela 1), indica teores elevados de isômeros trans em margarinas brasileiras e canadenses. Por outro lado, são observados níveis reduzidos de AGT nas margarinas avaliadas por Wagner et al..$^{44} \mathrm{e}$ Torres et al. ${ }^{45}$. Contudo, foram verificados níveis elevados de ácidos graxos saturados nestas margarinas, com valores médios próximos de 36\%. Esta estratégia industrial, reduz a razão entre os ácidos graxos polinsaturados e os ácidos graxos saturados (AGPI/ AGS), contribuindo para o aumento dos níveis plasmáticos de colesterol e triacilgliceróis, e elevando o risco de doenças cardiovasculares. Estratégias mais eficientes, combinando a hidrogenação parcial e a interesterificação química tem resultado na redução significativa de AGT, sem grandes alterações na relação AGPI/AGS. Com o desenvolvimento da interesterificação enzimática tem sido possível a produção de margarinas livres de isômeros trans ${ }^{49}$.

Os teores de AGT em batatas fritas analisadas por Santana et al. ${ }^{39}$ e Chiara et al. ${ }^{40} \mathrm{e}$ comparados com os dados obtidos por Innis et al. ${ }^{48}$, sugerem que este produto brasileiro apresenta níveis mais elevados de isômeros trans. Os dados relatados por Lake et al. ${ }^{43}$ também indicam isto, entretanto estão associados com a presença elevada de ácidos graxos saturados.

A comparação dos dados de AGT em gorduras hidrogenadas estudadas por Basso et al. ${ }^{2}$, Azevedo ${ }^{37}$ e Enig et al. ${ }^{46}$ através da análise por cromatografia gasosa, evidencia os teores mais 
elevados em gorduras brasileiras. Esta diferença pode ser justificada pela defasagem em cerca de vinte anos entre o início da produção de gordura hidrogenada no Brasil e nos Estados Unidos, que se reflete atualmente nas estratégias tecnológicas da indústria nacional. A análise dos teores de
AGT em gorduras brasileiras, avaliadas por espectrofotometria no infravermelho, também sugere esta condição, ao indicar que a produção de gorduras na última década sofreu poucas alterações que resultassem na redução dos isômeros trans.

Tabela 1. Teores de ácidos graxos trans em gorduras hidrogenadas e alimentos industrializados.

\begin{tabular}{|c|c|c|c|}
\hline & Produto & Teores médios de AGT & Referência \\
\hline \multirow{8}{*}{ Brasil } & Margarina sólida $(12)^{* 1}$ & $32,2(25,0-42,9) * * 1 a$ & \\
\hline & Margarina cremosa $(21)^{1}$ & $20,7 \quad(14,4-31,3)^{1 a}$ & \\
\hline & Gordura hidrogenada $(3)^{1}$ & $39,7 \quad(37,8-42,3)^{1 a}$ & ${ }^{1}$ Soares \& Franco $(1990)^{38}$ \\
\hline & Gordura hidrogenada $(12)^{2}$ & $29,1 \quad(0-53,9)^{2 a} ; 27,9(0-50,4)^{2 e}$ & ${ }^{2}$ Basso et al. $(1999)^{2}$ \\
\hline & Gordura hidrogenada $(28)^{3}$ & $34,9 \quad(9,5-54,6)^{3 a} ; 29,6(8,9-44,1)^{3 b}$ & ${ }^{3}$ Azevedo $(1999)^{37}$ \\
\hline & Batata frita $(25)^{4}$ & $10,42(3,4-21,1)^{4 b} ; 3,8(1,5-7,9)^{4 c}$ & ${ }^{4}$ Santana et al. (1999) ${ }^{39}$ \\
\hline & Batata frita $(18)^{5}$ & $2,50^{5 c}$ & ${ }^{5}$ Chiara \& Sichieri $(2001)^{40}$ \\
\hline & Biscoitos cracker e cookies $(12)^{5}$ & $2,80^{5 c}$ & \\
\hline \multirow[b]{2}{*}{ Argentina } & Margarina (3) & $18,2-31,8^{b}$ & Tavella et al. $(2000)^{41}$ \\
\hline & Biscoitos cracker e cookies (18) & $2,9-29,0^{b}$ & \\
\hline \multirow[t]{2}{*}{ Austrália } & Margarina (13) & $13,1 \quad(9,2-16,3)^{a} ; 12,2(8,0-14,5)^{b}$ & Mansour \& Sinclair $(1993)^{42}$ \\
\hline & Margarina (7) & $16,4 \quad(12,6-19,7)^{\mathrm{e}}$ & \\
\hline \multirow[t]{2}{*}{ Nova Zelândia } & Batata frita (2) & $5,6 \quad(5,4-5,8)^{\mathrm{e}}$ & Lake et al. (1998) ${ }^{43}$ \\
\hline & Biscoitos cracker (5) & $2,0 \quad(1,2-3,9)^{b}$ & \\
\hline Áustria & Margarina (9) & $1,6 \quad(0,3-3,73)^{b}$ & Wagner et al. $(2000)^{44}$ \\
\hline \multirow[t]{3}{*}{ Portugal } & Margarina (10) & $3,0 \quad(0,2-8,9)^{b}$ & Torres et al. $(2000)^{45}$ \\
\hline & Margarina sólida $(24)^{1},(60)^{2}$ & $22,4 \quad(15,9-31,0)^{1 d} ; 21,7(14,8-30,1)^{2 b}$ & \\
\hline & Margarina cremosa $(13)^{1},(26)^{2}$ & $12,7 \quad(6,8-17,6)^{1 d} ; 15,1(10,7-21,0)^{2 b}$ & \\
\hline \multirow[t]{5}{*}{ EUA } & Gordura hidrogenada $(07)^{1}$ & $21,71(8,7-35,4)^{1 d}$ & ${ }^{1}$ Enig et al. $(1983)^{46}$ \\
\hline & Biscoitos cracker $(20)^{1}$ & $10,9 \quad(1,9-29,0)^{1 d}$ & ${ }^{2}$ Slover et al. (1985)47 \\
\hline & Biscoitos cookies $(25)^{1}$ & $16,7 \quad(2,5-34,2)^{1 d}$ & \\
\hline & Margarina sólida $(14)^{1},(4)^{2}$ & $39,8 \quad(31,1-44,6)^{1 \mathrm{~b}} ; 35,9(30,7-42,2)^{2 \mathrm{a}}$ & \\
\hline & Margarina cremosa $(14)^{1},(5)^{2}$ & $16,8 \quad(1,1-44,4)^{1 b} ; 16,1(12,4-19,5)^{2 a}$ & ${ }^{1}$ Innis et al. $(1999)^{48}$ \\
\hline \multirow[t]{3}{*}{ Canadá } & Batata frita $(6)^{1}$ & $5,9 \quad(0,4-25,3)^{1 \mathrm{~b}} ; 1,4(0,1-5,7)^{1 \mathrm{c}}$ & ${ }^{2}$ Ratnayake et al. $(1990)^{31}$ \\
\hline & Biscoitos cracker (14) ${ }^{1}$ & $40,3 \quad(23,5-51,3)^{1 b} ; 6,4(0,7-12,9)^{1 c}$ & \\
\hline & Biscoitos cookies $(19)^{1}$ & $23,0 \quad(1,4-45,7)^{1 b} ; 3,5(0,3-8,1)^{1 c}$ & \\
\hline
\end{tabular}

(*) número de amostras avaliadas; ${ }^{(* *)}$ intervalo dos valores medidos.

(a) determinado por espectrofotometria no infravermelho, com valor em porcentagem de ésteres metílicos de ácidos graxos; (b) determinado por cromatografia gasosa, com valor em porcentagem de ésteres metílicos de ácidos graxos; ${ }^{(c)}$ determinado por cromatografia gasosa, com valor em g/100g do produto; (d) determinado por CCD/Ag+ associada a cromatografia gasosa, com valor em porcentagem de ésteres metílicos de ácidos graxos; $^{(e)}$ determinado por cromatografia gasosa em associação com espectrofotometria no infravermelho, com valor em porcentagem de ésteres metílicos de ácidos graxos. 


\section{O N CLUS Ã O}

Conforme as implicações nutricionais abordadas, a ingestão elevada de ácidos graxos trans contribui para o aumento do risco de doenças cardiovasculares.

A comparação entre os teores de ácidos graxos trans em gorduras hidrogenadas, margarinas e batatas frita produzidos em vários países, incluindo o Brasil, indica a presença elevada destes ácidos graxos em produtos brasileiros. Estes dados sugerem a ingestão elevada de isômeros trans pela população brasileira.

A redução do consumo de ácidos graxos trans no Brasil deve envolver a ampla divulgação dos seus malefícios à população e a realização de mais estudos que visem determinar o seu conteúdo nos alimentos e estimar os níveis de ingestão diária. Além disso, ações governamentais devem incentivar o desenvolvimento de tecnologias que possibilitem a produção de gorduras com níveis reduzidos de isômeros trans sem elevar o conteúdo de ácidos graxos saturados.

\section{A GRADECIMENTOS}

A CAPES pela suporte financeiro.

\section{REF ER Ê N CIAS}

1. Feldman EB, Krisetherton $P$, Kritchevsky $D$, Lichtenstein $\mathrm{AH}$. Position paper on trans fatty acids. Am J Clin Nutr 1996; 63(5):663-70.

2. Basso R, Almeida IG, Mancini JF. Avaliação qualitativa e quantitativa dos ácidos graxos trans em gorduras vegetais hidrogenadas. Bol SBCTA 1999; 33(1):57-63.

3. Anderson JT, Grande F, Keys A. Hidrogenated fats in the diet and lipids in the serum man. J Nutr 1961; 75(4):388-94

4. Katan MB, Mensink RP, Zock PL. Trans fatty acids and their effect on lipoproteins in humans. Annu Rev Nutr 1995; 15(5):473-93.
5. Mensink RP, Katan MB. Effect of dietary trans fatty acids on high-density and low-density lipoprotein cholesterol levels in healthy subjects. N Engl J Med 1990; 323(7):439-45.

6. Zock PL, Katan MB. Hydrogenation alternatives: effects of trans fatty acids and stearic acids versus linoleic acid on serum lipids and lipoproteins in humans. J Lipid Res 1992; 33(10):399-410.

7. Judd JT, Clevidence BA, Muesing RA, Wittes J, Sunkin ME, Podczasy JJ. Dietary trans fatty acids: effects of plasma lipids and lipoproteins on healthy men and women. Am J Clin Nutr 1994; 59(4): 861-8.

8. Aro $A$, Jauhiainen $M$, Partanen $R$, Salminen I, Mutanen M. Stearic acid, trans fatty acids, and dairy fat: effects on serum and lipoprotein, lipids apolipoproteins, lipoprotein(a), and lipid transfer proteins in health subjects. Am J Clin Nutr 1997; 65(5):1419-26.

9. Sundram K, Ismail A, Hayes KC, Jeyamalar R, Pathmanathan R. Trans (elaidic) fatty acids adversely affect the lipoprotein profile relative to specific satured fatty acids in humans. J Nutr 1997; 127(3):514s-20s.

10. Muller H, Jordal O, Seljeflot I, Kierulf P, Kirkhus E, Ledsaak $O$, et al. Effect on plasma lipids and lipoproteins of replacing partially hydrogenated fish oil with vegetable fat in margarine. Br J Nutr 1998; 80(3):243-51.

11. Lichtenstein AH, Ausman LM, Jalbert SM, Schaefer EJ. Effects of different forms of dietary hydrogenated fats on serum lipoprotein cholesterol levels. N Engl J Med 1999; 340(25): 1933-40.

12. Ascherio A, Katan MB, Zock, PL, Stampfer, MJ, Willett WC. Trans fatty acids and coronary heart disease. N Engl J Med 1999; 340(25):1994-8.

13. Stampfer MJ, Sacks, FM, Salvini S, Willett WC, Hennekens CH. A prospective-study of cholesterol, apolipoproteins, and the risk of myocardial-infarction. N Engl J Med 1991; 325(6):373-81.

14. Hu FB, Manson JE, Willett WC. Types of dietary fat and risk of coronary heart disease: a critical review. J Am Coll Nutr 2001; 20(1):5-19. 
15. Nestel P, Noakes M, Belling B, Mcarthur R, Clinfton $P$, Janus $E$, et al. Plasma lipoprotein lipid and Lp[a] changes with substitution of elaidic acid for oleic acid in the diet. J Lipid Res 1992; 33(78):1029-36.

16. Mensink RP, Zock PL, Katan MB, Hornstra G. Effect of dietary cis and trans fatty acids on serum lipoprotein[a] levels in humans. J Lipid Res 1992; 33(10):1493-501.

17. Lichtenstein AH, Ausman LM, Carrasco W, Jenner JL, Ordovas JM, Schaefer EJ. Hydrogenation impairs the hypolipidemic effect of corn oil in humans, trans fatty acids and plasma-lipids. Arterioscler Thromb 1993; 13(2):154-61.

18. Lippi G, Guidi G. Biochemical risk factors and patient's outcome: The case of lipoprotein(a). Clin Chim Acta 1999; 280(1):59-71.

19. Dahlen GH. Lp(a) lipoprotein in cardiovascular disease. Atherosclerosis 1994; 108(2):111-26.

20. Hornstra GA, van Houwelingen AC, Kester DM, Sundram K. A palm-oil-enriched diet lowers serum lipoprotein[a] in normocholesterolemic volunteers. Atherosclerosis 1991; 90(1):91-3.

21. Judd JT, Baer DJ, Clevidence BA, Muesing RA, Chen SC, Weststrat JA, et al. Effects of margarine compared with those of butter on blood lipid profiles related to cardiovascular disease risk factors in normolipemic adults fed controlled diets. Am J Clin Nutr 1998; 68(4):768-77.

22. Kirstein D, Hoy CE, Holmer G. Effect of dietary fats on the delta-6-desaturation and delta-5-desaturation of fatty-acids in rat-liver microsomes. Br J Nutr 1983; 50(3):749-53.

23. Mahfouz MM, Smith TL, Kummerow, FA. Effect of dietary fats on desaturase activities and the biosynthesis of fatty-acids in rat-liver microsomes. Lipids 1984; 19(3):214-22.

24. Blond JP, Henchiri C, Precigou P, Grandgirar DA, Sebedio JL. Effect of 18-3 n-3 geometrical-isomers of heated linseed oil on the biosynthesis of arachidonic-acid in rat. Nutr Res 1990; 10(1): 69-79.

25. Decsi T, Koletzko B. Do trans fatty acids impair linoleic acid metabolism in children? Ann Nutr Metab 1995; 39(1):36-41.
26. Scrimgeour CM, Macvean A, Fernie C, Sebedio JL, Riemersma AR. Dietary trans linolenic acid does not inhibit and desaturation of linoleic acid in man. Eur J Lipid Sci Technol 2001; 103(6):341-349.

27. Ledoux M, Laloux L, Wolff R. Analytical methods for determination of trans-C 18 fatty acids isomers in milk fat. A review. Analusis 2000; 28(5):402-12.

28. Seppanen-Laakso T, Laakso I, Hiltunen R. Analysis of fatty acids by gas chromatography, and its relevance to research on health and nutrition. Anal Chim Acta 2002, 465(1-2):39-62.

29. Christie WW. Gas chromatography and lipids. Glasgow: The Oily Press; 1994. 307p.

30. Bysted A, Cold S, Holmer G. An optimized method for fatty acid analysis, including quantification of trans fatty acids, in human adipose tissue by gas-liquid chromatography. Scand J Clin Lab Invest 1999; 59(3):205-14.

31. Ratnayake WMN, Hollywood R, O'Grady E, Beare-Rogers JL. Determination of cis and trans-octadecenoic acids in margarines by gas liquid chromatography-infrared espectrophotometry. J Am Oil Chem Soc 1990; 67(11):804-10.

32. Favier JP, Bicanic D, van de Bovenkamp $P$, Chirtoc M, Helander P. Detection of total trans fatty acids content in margarine: an intercomparison study of GLC, GLC+TLC, FT-IR, and optothermal window (open photoacoustic cell). Anal Chem 1996; 68(5):729-33.

33. Ratnayake WMN. Determination of trans unsaturation by infrared spectrophotometry and determination of fatty acid composition of partially hidrogenated vegetable oils and animals fats by gas chromatography/infrared spectrofotmetry: collaborative study. J Assoc Off Anal Chem 1995; 78(3):783-802.

34. Larque E, Zamora S, Gil A. Dietary trans fatty acids in early life: a review. Early Hum Dev 2001; 65(2):31s-41s.

35. Aro A, van Amelsvoort Becker W, van Erp-Baart MA, Kafatos A, Leth T, van Poppel, G. Trans fatty acids in dietary fats and oils from 14 European Countries: The TRANSFAIR study. J. Food Comp Anal 1998; 11(2):137-49. 
36. Dionisi F, Golay PA, Fay LB. Influence of milk fat presence on the determination of trans fatty acids in fats used for infant formulae. Anal Chim Acta 2002; 465(1):395-407.

37. Azevedo CH. Teores de isômeros trans em gorduras vegetais hidrogenadas avaliadas por diferentes técnicas instrumentais [dissertação]. Campinas: Faculdade de Engenharia de Alimentos, Unicamp; 1999.

38. Soares LMV, Franco MRB. Níveis de trans-isômeros e composição de ácidos graxos de margarinas e produtos hidrogenados semelhantes. Ciênc Tecnol Aliment 1990; 10(1):57-71.

39. Santana DMN, Marques MM, Rosa CAR. Determinação por cromatografia gasosa da composição em ácidos graxos e teor de ácido trans oléico em algumas marcas de batata frita. Bol SBCTA 1999, 33(1):64-9.

40. Chiara VL, Sichieri R. Food consumption of adolescents. A simplified questionnaire for evaluating cardiovascular risk. Arq Bras Cardiol 2001; 77(4):337-41.

41. Tavella M, Peterson G, Espeche M, Cavallero E, Cipolla L, Perego L, Caballero B. Trans fatty acid content of a selection of foods in Argentina. Food Chem 2000; 69(2):209-13.

42. Mansour MP, Sinclair AJ. The trans fatty acid and positional (sn-2) fatty acid composition of some Australian margarines, dairy blends and animal fats. Asia Pac J Clin 1993; 4(2):155-63.
43. Lake R, Thomson B, Devane G, Scholes P. Trans fatty acid content of selected New Zealand Foods. J Food Comp Anal 1996; 9(4):365-74.

44. Wagner KH, Auer E, Elmadfa I. Content of trans fatty acids in margarines, plant oils, fried products and chocolate spreads in Austria. Eur Food Res and Technol 2002; 214(2):208-11.

45. Torres D, Casal S, Oliveira MBPP. Fatty acid composition of Portuguese spreadable fats with emphasis on trans isomers. Eur Food Res and Technol 2000; 210(4):237-41.

46. Enig MG, Pallansch LA, Sampugna J, Keeney M. Fatty acid composition of the fat in selected food items with emphasis on trans components. J Am Oil Chem Soc 1983; 60(10):1788-95.

47. Slover HT, Thompson JR, Davis CS, Merola GV. Lipids in margarines and margarine-like foods. J Am Oil Chem Soc 1990; 62(4):775-86.

48. Innis SM, Green TJ, Halsey TK. Variability in the trans fatty acid content of foods within a food category: implications for estimation of dietary trans fatty acids intakes. J Am Coll Nutr 1999; 18(3):255-60.

49. Katan MB. Exit trans fatty acids. Lancet 1995; 346:1245-6.

Recebido para publicação em 18 de fevereiro e aceito em 1 de outubro de 2003. 